\title{
Highly Efficient, Reversible Addition of Activated Methylene Compounds to Styrene Derivatives Catalyzed by Silver Catalysts
}

\author{
Xiaoquan Yao and Chao-Jun Li* \\ Department of Chemistry, McGill University, 801 Sherbrooke St. West, Montreal, Quebec H3A 2K6, Canada \\ Email: cj.li@,McGill.ca
}

Table of Contents

General Experimental Methods

Characterization for compounds $\mathbf{5 c}, \mathbf{5 d}, \mathbf{5 f}, \mathbf{6 c}, \mathbf{6 d}, \mathbf{7 a}$ and $\mathbf{7 b}$

Reference

${ }^{1} \mathrm{H}$ NMR spectra compounds 5c, 5d, 5f, 6c, 6d, 7a and 7b
S2

S2 to S5

S5

S6 to S13 


\section{Experimental Section}

All experiments were carried out under an inert atmosphere of nitrogen. Nitromethane and DCE were dried by $4 \AA \AA$ molecular sieves. Flash column chromatography was performed over silica gel $30-60 \mu \mathrm{m} .{ }^{1} \mathrm{H}$ NMR and ${ }^{13} \mathrm{C}$ NMR spectra were acquired by $400 \mathrm{MHz}$ and 100 $\mathrm{MHz}$, or $300 \mathrm{MHz}$ and $75 \mathrm{MHz}$, respectively, and referenced to the internal solvent signals. Compounds 3a-3o and $\mathbf{4}$ have been reported. ${ }^{1,2}$ Compounds 5a-5f were prepared following the reported method. ${ }^{3,4}$ Compound $5 \mathbf{a}-\mathbf{5 b}, \mathbf{5 e}$ and $\mathbf{6 a - 6 \mathbf { b }}$ have also been reported. ${ }^{3,4}$ The data for new compounds $\mathbf{5 c}, \mathbf{5 d}, \mathbf{5 f}, \mathbf{6 c}, \mathbf{6 d}, \mathbf{7 a}$ and $\mathbf{7 b}$ were described here.

Typical procedure (entry 1, Table 2): AgOTf $(25.6 \mathrm{mg}, 0.1 \mathrm{mmol})$ were added into $2 \mathrm{~mL}$ of nitromethane under $\mathrm{N}_{2} .2$,4-Pentanedione $(103 \mu \mathrm{L}, 1 \mathrm{mmol})$ and styrene $(172 \mu \mathrm{L}, 1.5 \mathrm{mmol})$ were introduced into the solution. The mixture was stirred and heated to $100^{\circ} \mathrm{C}$ overnight and removed solvent under reduced pressure. The reside was isolated by flash chromatography with silica to give diketone alkylation product with $76 \%$ yield.

2-(5-Phenyl-pent-4-enoyl)-cyclopentanone (1:1 mixture of enol and diketone) (5c). IR (liquid film): $v_{\max } 3084,3023,2963,2919,2854,1741,1708,1653,1612,1446,1372,1230$, $1152,963 \mathrm{~cm}^{-1} ;{ }^{1} \mathrm{H}$ NMR $\left(\mathrm{CDCl}_{3}, 400 \mathrm{MHz}, \mathrm{ppm}\right) \delta 7.33-7.28(\mathrm{~m}, 4 \mathrm{H}), 7.22-7.17(\mathrm{~m}, 1 \mathrm{H})$, 6.45-6.39(two d peaks overlap, 1H), 6.25-6.16(two td peaks overlap, $1 \mathrm{H}$ ), 3.99(t, $J=8.0 \mathrm{~Hz}$, $0.5 \mathrm{H}$, from diketone), $3.06(\mathrm{dt}, J=17.6,7.2 \mathrm{~Hz}, 0.5 \mathrm{H}$, from enol), $2.67(\mathrm{dt}, J=17.6,7.2 \mathrm{~Hz}$, 0.5H, from enol), 2.59-2.04(m, 6H), 2.31-2.25(m, 1H), 2.07(m, 1H), 2.07(m, 1H), 1.951.84(m, 2H); ${ }^{13} \mathrm{C} \mathrm{NMR}\left(\mathrm{CDCl}_{3}, 100 \mathrm{MHz}, \mathrm{ppm}\right) \delta 213.1,204.9,203.8,178.4,137.6,137.5$, $131.2,130.9,129.0,128.8,128.7,128.7127 .4127 .3126 .2,126.2,110.0,62.4,42.9,39.2$, 37.2, 34.8, 29.2, 27.1, 26.1, 25.7, 21.8, 20.8; MS (EI) $m / z(\%) 242\left(\mathrm{M}^{+}\right), 224,151,130$, 117(100), 91; HRMS calcd for $\mathrm{C}_{16} \mathrm{H}_{18} \mathrm{O}_{2}$ : 242.1307; found: 242.1304 . 
1,7-Diphenyl-hept-6-ene-1,3-dione (5d). IR (liquid film): $v_{\max }$ 3063, 3025, 2914, 1720, $1673,1602,1571,1494,1267,1142,963 \mathrm{~cm}^{-1} ;{ }^{1} \mathrm{H} \mathrm{NMR}\left(\mathrm{CDCl}_{3}, 400 \mathrm{MHz}, \mathrm{ppm}\right) \delta 7.89(\mathrm{~d}, J$ $=8 \mathrm{~Hz}, 2 \mathrm{H}), 7.53-7.21(\mathrm{~m}, 8 \mathrm{H}), 6.48(\mathrm{~d}, J=16 \mathrm{~Hz}, 1 \mathrm{H}), 6.28-6.22(\mathrm{~m}, 2 \mathrm{H}), 2.64-2.63(\mathrm{~m}, 4 \mathrm{H})$; ${ }^{13} \mathrm{C}$ NMR $\left(\mathrm{CDCl}_{3}, 75 \mathrm{MHz}, \mathrm{ppm}\right) \delta 196.1,183.3,137.5,135.0,132.5,131.2,128.9,128.8$, 127.4, 127.2, 126.3, 96.6, 39.4, 29.4; MS (EI) $m / z(\%)$ 278( $\left.{ }^{+}\right), 260,187,173,147,130$, 105(100), 91, 77; HRMS calcd for $\mathrm{C}_{19} \mathrm{H}_{18} \mathrm{O}_{2}: 278.1307$; found: 278.1302 .

1-Phenyl-non-6-ene-1,3-dione (5f). IR (liquid film): $v_{\max }$ 3065, 2967, 2931, 2875, 1621, $1575,1491,1459,1301,1269,1181,1075,966 \mathrm{~cm}^{-1} ;{ }^{1} \mathrm{H} \mathrm{NMR}\left(\mathrm{CDCl}_{3}, 300 \mathrm{MHz}, \mathrm{ppm}\right) \delta$ 7.90-7.86(m, 2H), 7.54-7.41(m, 3H), 6.17(s, 1H), 5.49(m, 2H), 2.53-2.35(m, 4H), 2.02(m, $2 \mathrm{H}), 0.98(\mathrm{t}, J=6.9 \mathrm{~Hz}, 3 \mathrm{H}) ;{ }^{13} \mathrm{C} \mathrm{NMR}\left(\mathrm{CDCl}_{3}, 75 \mathrm{MHz}, \mathrm{ppm}\right) \delta 196.4,183.4,135.2,133.6$, $132.4,128.8,127.3,127.2,96.5,39.7,29.0,26.0,14.3$; MS (EI) $m / z(\%) 230\left(\mathrm{M}^{+}\right), 201,162$, 147, 120, 105(100), 69; HRMS calcd for $\mathrm{C}_{15} \mathrm{H}_{18} \mathrm{O}_{2}: 230.1307$; found: 230.1300.

10-Phenyl-spiro[4.5]decane-1,6-dione (6c). IR (liquid film): $v_{\max }$ 3060, 3030, 2985, 2880, $1748,1694,1493,1449,1316,1251,1155,1130,1098,980,962 \mathrm{~cm}^{-1} ;{ }^{1} \mathrm{H} \mathrm{NMR}\left(\mathrm{CDCl}_{3}\right.$, $300 \mathrm{MHz}, \mathrm{ppm}) \delta \quad 7.29-7.10(\mathrm{~m}, 5 \mathrm{H}), 3.57(\mathrm{dd}, J=9.9,4.2 \mathrm{~Hz}, 1 \mathrm{H}), 2.60-2.56(\mathrm{~m}, 2 \mathrm{H}), 2.27-$ 2.19(m, 3H), 2.08-1.68(m, 6H), 1.45-1.40(m, 1H); ${ }^{13} \mathrm{C} \mathrm{NMR}\left(\mathrm{CDCl}_{3}, 75 \mathrm{MHz}, \mathrm{ppm}\right) \delta 216.7$, $210.3,140.5,129.2,128.5,127.1,68.8,48.1,39.5,39.7,30.5,27.9,23.8,19.5 ; \mathrm{MS}(\mathrm{EI}) \mathrm{m} / z$ (\%) 242( $\left.\mathrm{M}^{+}, 100\right), 224,196,185,171,137,129,115,104,91,81$; HRMS calcd for $\mathrm{C}_{16} \mathrm{H}_{18} \mathrm{O}_{2}$ : 242.1307; found: 242.1310 .

another diastereomer: IR (liquid film): $v_{\max }$ 3062, 3030, 2956, 2921, 2864, 1729, 1699, 1494, 1453, 1444, 1319, 1136, 1118, 1013, 927, 906, 896 $\mathrm{cm}^{-1} ;{ }^{1} \mathrm{H}$ NMR $\left(\mathrm{CDCl}_{3}, 300 \mathrm{MHz}\right.$, ppm) $\delta$ 7.29-7.14(m, 5H), 3.11(td, $J=13.5,6.3 \mathrm{~Hz}, 1 \mathrm{H}), 2.95-2.89(\mathrm{~m}, 1 \mathrm{H}), 2.82-2.73(\mathrm{~m}$, $2 \mathrm{H}), 2.51-2.45(\mathrm{~m}, 1 \mathrm{H}), 2.26-2.04(\mathrm{~m}, 2 \mathrm{H}), 1.86-1.42(\mathrm{~m}, 5 \mathrm{H}), 1.44-1.07(\mathrm{~m}, 1 \mathrm{H}) ;{ }^{13} \mathrm{C} \mathrm{NMR}$ $\left(\mathrm{CDCl}_{3}, 75 \mathrm{MHz}, \mathrm{ppm}\right) \delta 216.3,205.6,141.3,128.9,128.6,70.2,55.7,41.6,39.3,30.1,28.5$, 
26.2, 19.8; MS (EI) $m / z(\%) \quad 242\left(\mathrm{M}^{+}, 100\right), 224,196,185,171,137,129,115,104,91,81$; HRMS calcd for $\mathrm{C}_{16} \mathrm{H}_{18} \mathrm{O}_{2}$ : 242.1307; found: 242.1309 .

2-Benzoyl-3-phenyl-cyclohexanone (6d). IR (liquid film): $v_{\max }$ 3061, 3030, 2951, 2941, $2920,2862,1706,1675,1596,1446,1351,1241,1170,1103,1015,916 \mathrm{~cm}^{-1} ;{ }^{1} \mathrm{H}$ NMR $\left(\mathrm{CDCl}_{3}, 300 \mathrm{MHz}, \mathrm{ppm}\right) \delta 7.71(\mathrm{~d}, J=7.2 \mathrm{~Hz}, 2 \mathrm{H}), 7.48(\mathrm{~m}, 1 \mathrm{H}), 7.36(\mathrm{~m}, 2 \mathrm{H}), 7.25-7.21(\mathrm{~m}$, 4H), 7.17-7.11(m, 1H), 4.72(d, $J=10.8 \mathrm{~Hz}, 1 \mathrm{H}), 3.72(\mathrm{ddd}, J=10.8,10.8,3.6 \mathrm{~Hz}, 1 \mathrm{H}), 2.64-$ 2.59(m, 2H), 2.21-2.15(m, 2H), 2.08-1.91(m, 2H); ${ }^{13} \mathrm{C} \mathrm{NMR}\left(\mathrm{CDCl}_{3}, 75 \mathrm{MHz}, \mathrm{ppm}\right) \delta 207.9$, $197.3,143.0,137.8,133.2,128.9,128.7,128.3,127.3,127.0,64.1,47.4,42.3,33.4,25.4 ; \mathrm{MS}$ (EI) $m / z(\%) \quad 278\left(\mathrm{M}^{+}\right), 260,221,204,173(100), 105,91,77$; HRMS calcd for $\mathrm{C}_{19} \mathrm{H}_{18} \mathrm{O}_{2}$ : 278.1307; found: 278.1303 .

2-(5-Methyl-dihydro-furan-2-ylidene)-1-phenyl-ethanone (7a). IR (liquid film): $v_{\max } 3059$, $2976,2932,1656,1598,1584,1570,1440,1386,1187,1160,1040,910,893 \mathrm{~cm}^{-1} ;{ }^{1} \mathrm{H}$ NMR $\left(\mathrm{CDCl}_{3}, 400 \mathrm{MHz}, \mathrm{ppm}\right) \delta 7.90-7.88(\mathrm{~m}, 2 \mathrm{H}), 7.48-7.40(\mathrm{~m}, 3 \mathrm{H}), 6.50(\mathrm{~d}, J=1.6 \mathrm{~Hz}, 1 \mathrm{H})$, $4.62(\mathrm{ddq}, J=8.0,6.4,6.4 \mathrm{~Hz}, 1 \mathrm{H}), 3.51(\mathrm{dddd}, J=19.2,8.8,4,1.6 \mathrm{~Hz}, 1 \mathrm{H}), 3.16(\mathrm{dddd}, J=$ $19.2,9.6,9.6,2 \mathrm{~Hz}, 1 \mathrm{H}), 2.30(\mathrm{~m}, 1 \mathrm{H}), 1.78-1.69(\mathrm{~m}, 2 \mathrm{H}), 1.42(\mathrm{~d}, J=6.4 \mathrm{~Hz}, 3 \mathrm{H}) ;{ }^{13} \mathrm{C} \mathrm{NMR}$ $\left(\mathrm{CDCl}_{3}, 75 \mathrm{MHz}, \mathrm{ppm}\right) \delta 207.1,179.1,145.8,131.9,128.5,127.8,95.1,80.9,32.5,31.4$, 20.9; MS (EI) m/z (\%) 202(M $\left.\mathrm{M}^{+}, 100\right), 173,145,125,105,69$; HRMS calcd for $\mathrm{C}_{13} \mathrm{H}_{14} \mathrm{O}_{2}$ : 202.0994; found: 202.0992 .

The configuration of the double bond was determined by 1D NOESY (the proton on 6.50ppm was selected).

1-Phenyl-2-(5-propyl-dihydro-furan-2-ylidene)-ethanone (7b). IR (liquid film): $v_{\max } 3060$, $2958,2931,2873,1655,1599,1585,1571,1445,1380,1164,903 \mathrm{~cm}^{-1} ;{ }^{1} \mathrm{H} \mathrm{NMR}\left(\mathrm{CDCl}_{3}\right.$, $400 \mathrm{MHz}, \mathrm{ppm}) \delta 7.91-7.89(\mathrm{~m}, 2 \mathrm{H}), 7.48-7.40(\mathrm{~m}, 3 \mathrm{H}), 6.50(\mathrm{~s}, 1 \mathrm{H}), 4.48(\mathrm{dddd}, J=7.2,7.2$, 7.2, 7.2Hz, 1H), 3.49(dddd, $J=19.2,9.6,4,1.2 \mathrm{~Hz}, 1 \mathrm{H}), 3.14(\mathrm{dddd}, J=19.2,9.6,9.6,2 \mathrm{~Hz}$, $1 \mathrm{H}), 2.28(\mathrm{~m}, 1 \mathrm{H}), 1.80-1.70(\mathrm{~m}, 2 \mathrm{H}), 1.60-1.41(\mathrm{~m}, 4 \mathrm{H}), 1.00(\mathrm{t}, J=7.2,3 \mathrm{H}) ;{ }^{13} \mathrm{C} \mathrm{NMR}$ 
$\left(\mathrm{CDCl}_{3}, 75 \mathrm{MHz}, \mathrm{ppm}\right) \delta 190.5,179.3,140.1,131.8,128.5,127.8,95.0,84.7,37.5,32.3$, 29.7, 19.4, 14.4; MS (EI) $m / z(\%)$ 230(M $\left.{ }^{+}\right), 201,173,161,147,105(100), 77$; HRMS calcd for $\mathrm{C}_{15} \mathrm{H}_{18} \mathrm{O}_{2}$ : 230.1307; found: 230.1301 .

The five member ring and the configuration of the double bond were determined by $\mathrm{H}-\mathrm{H}$ COSY and 1D NOESY (the proton on 6.50ppm was selected).

\section{Reference:}

1 Yao, X. Q.; Li, C.-J. J. Am. Chem. Soc. 2004, 126, 6884

2 Tsucchimoto, T., Kamiyama, S.; Negoro, R.; Shirakawa, E.; Kawakami, Y. Chem. Commun. 2003,852

3 (a) Pei, T.; Widenhoefer, R. A. J. Am. Chem. Soc. 2001, 123, 11290; (b) Pei, T.;

Widenhoefer, R. A. Chem. Commun. 2002, 650; and the supporting information

4 (a) Huckin, S. N.; Weiler, L. J. Am. Chem. Soc. 1974, 96, 1082; (b) Snider, B. B.; Patricia, J. J. J. Org. Chem. 1989, 54, 3846 
y×a-z: 918

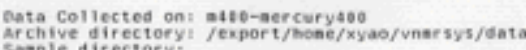

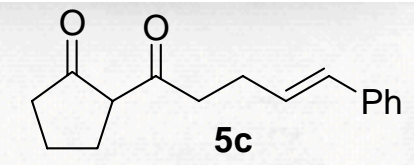

rille: parotow

Solvent: $\mathrm{CDCl}^{2}$, $298.1 \mathrm{~K}$

Relax gelay 1,000 sec

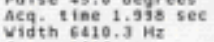

obstave ${ }^{41}, 400.1245662$ MHI

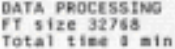

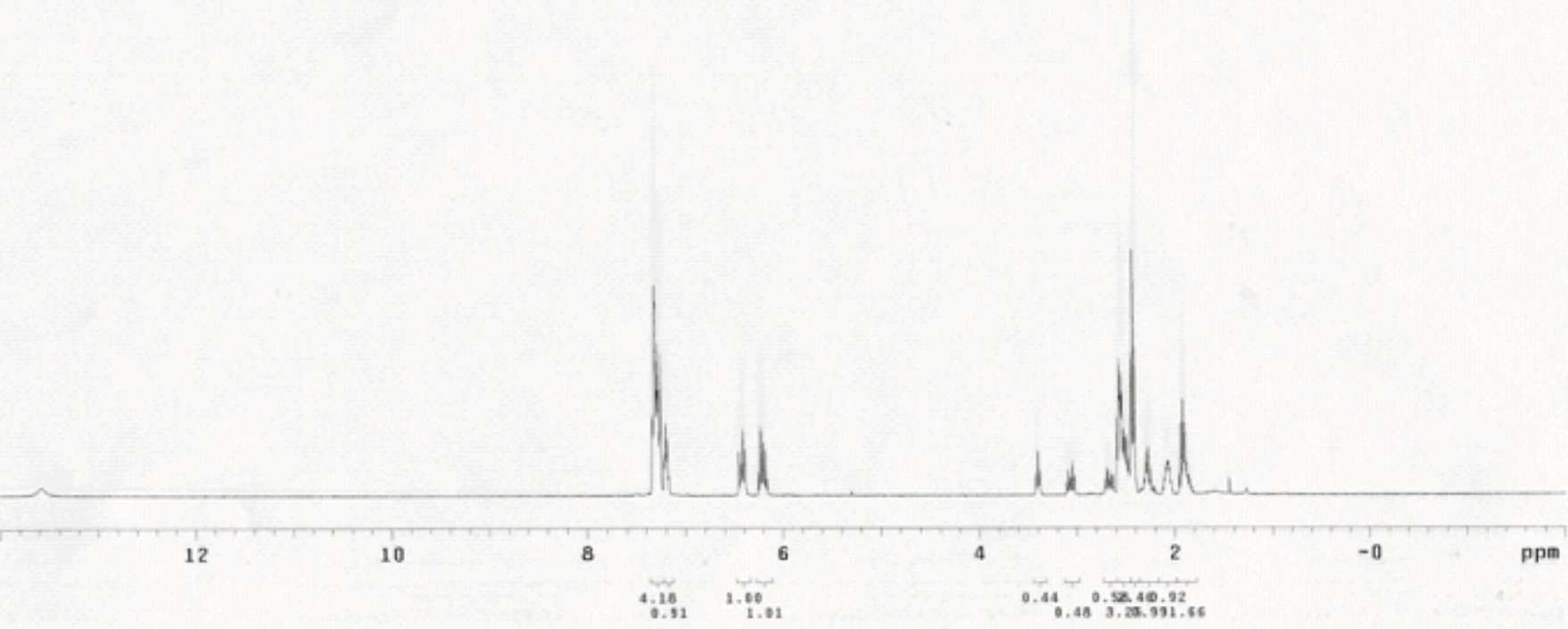




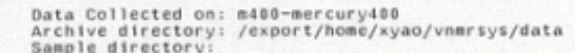

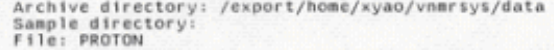

Pulse sequence: s2pul

Solvent: $\mathrm{CDCl}^{3}$, $29.1 \mathrm{~K}$
Temp. $25.0 \mathrm{C} / 298.1 \mathrm{~K}$

Relax. delay 1.000 sec
Pulse 45.0 degrees

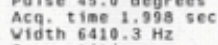

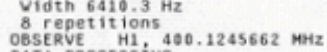

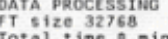
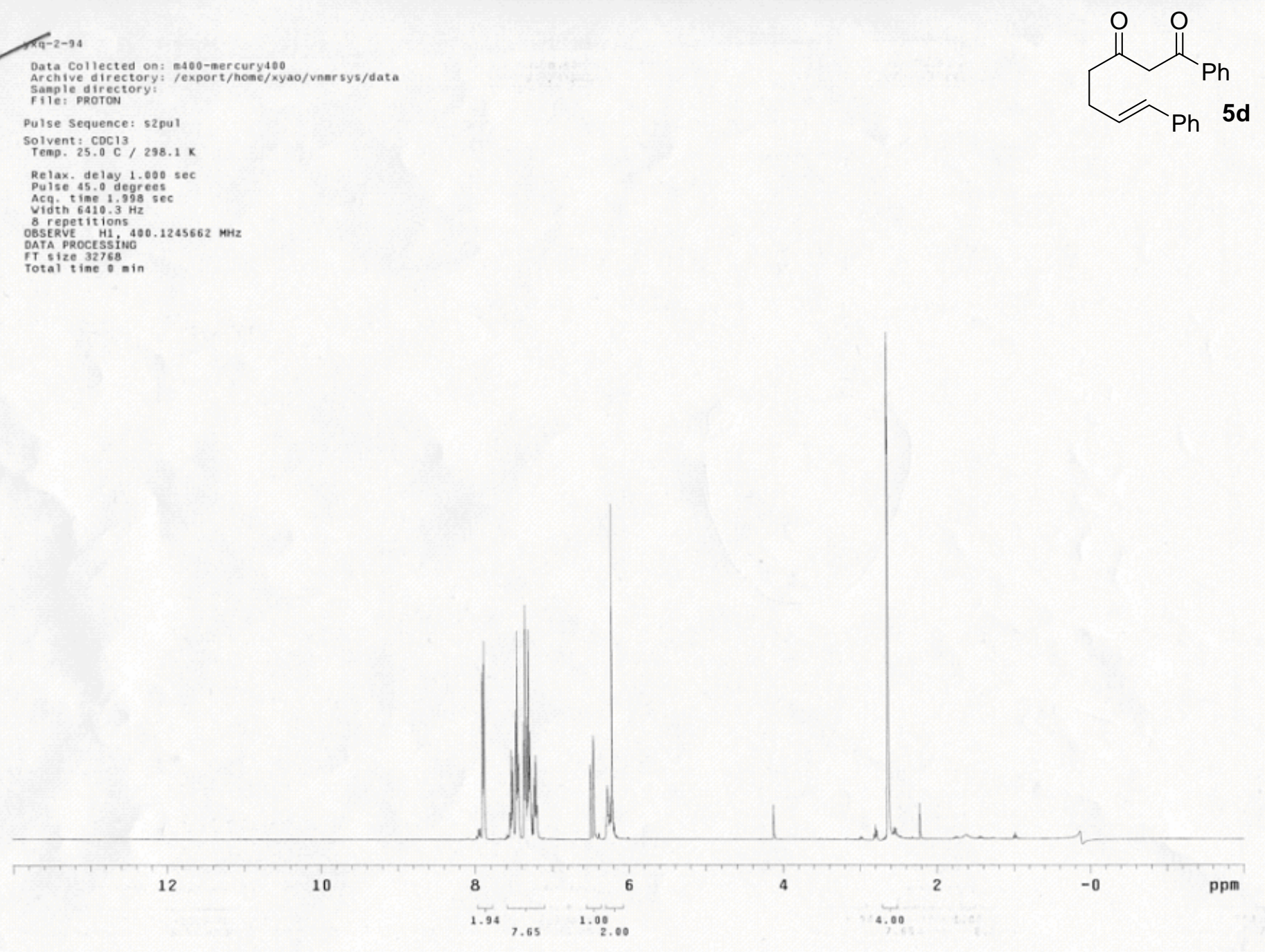
$y \times q-2-124$

Data collected on: 300 -mercury 300
Archive directory: /export/hooe/xyao/vanrsys/data

Archive directory:
Sanple directory:
ille: proron

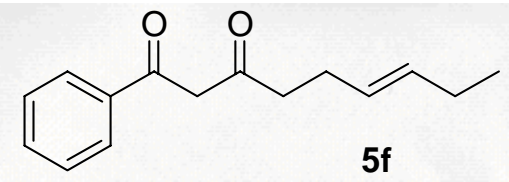

Relax, delay 1.000 sec

pelate

Acgith ${ }^{2} 4803.1998$

DESERVE H1, $300.0595500 \mathrm{MHZ}$

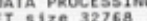

Total time 0 in

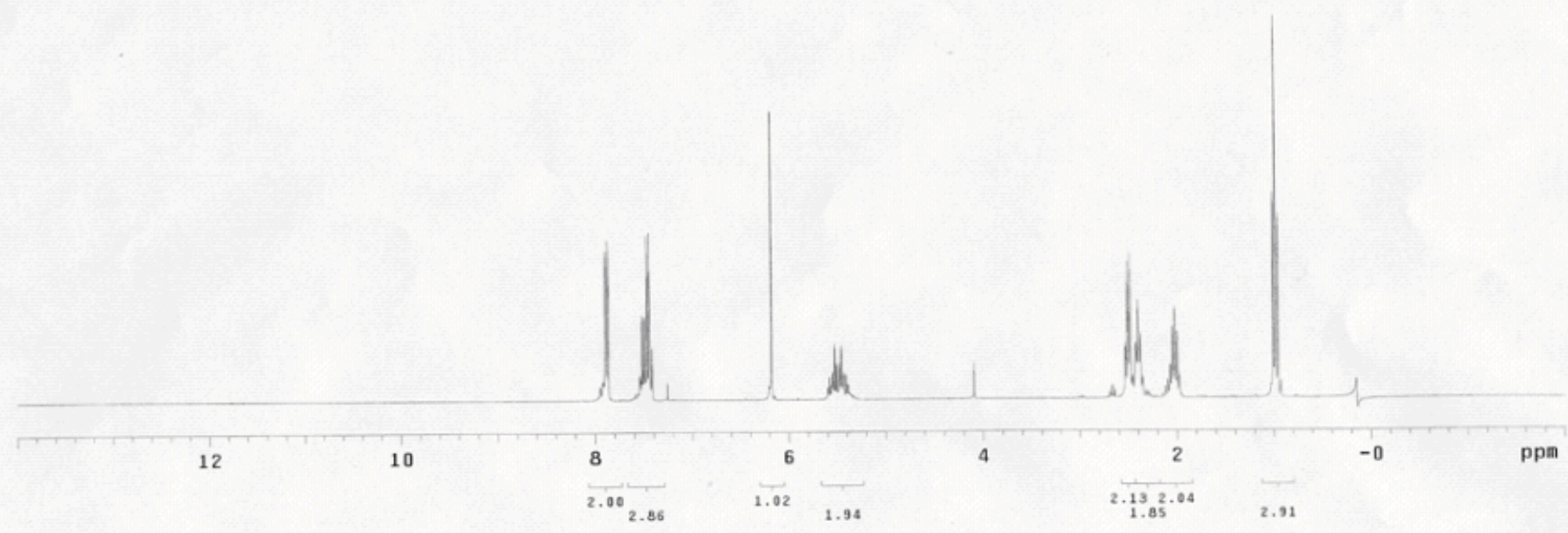


y×Q-2-93c-1uohuan

aata Collected on:
archo-nercury300
Archive directory: /export/home/xyao/vnnrsys/data

sample director

Pulse sequence: s2pu

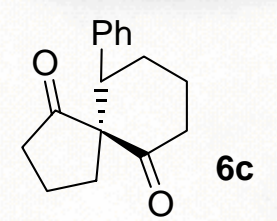

Relax. delay $1.000 \mathrm{sec}$

Acg. tine 1.938 sec

vidth $4803.1 \mathrm{~Hz}$

obstrve H1, $300.0595500 \mathrm{MHz}$

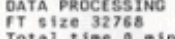

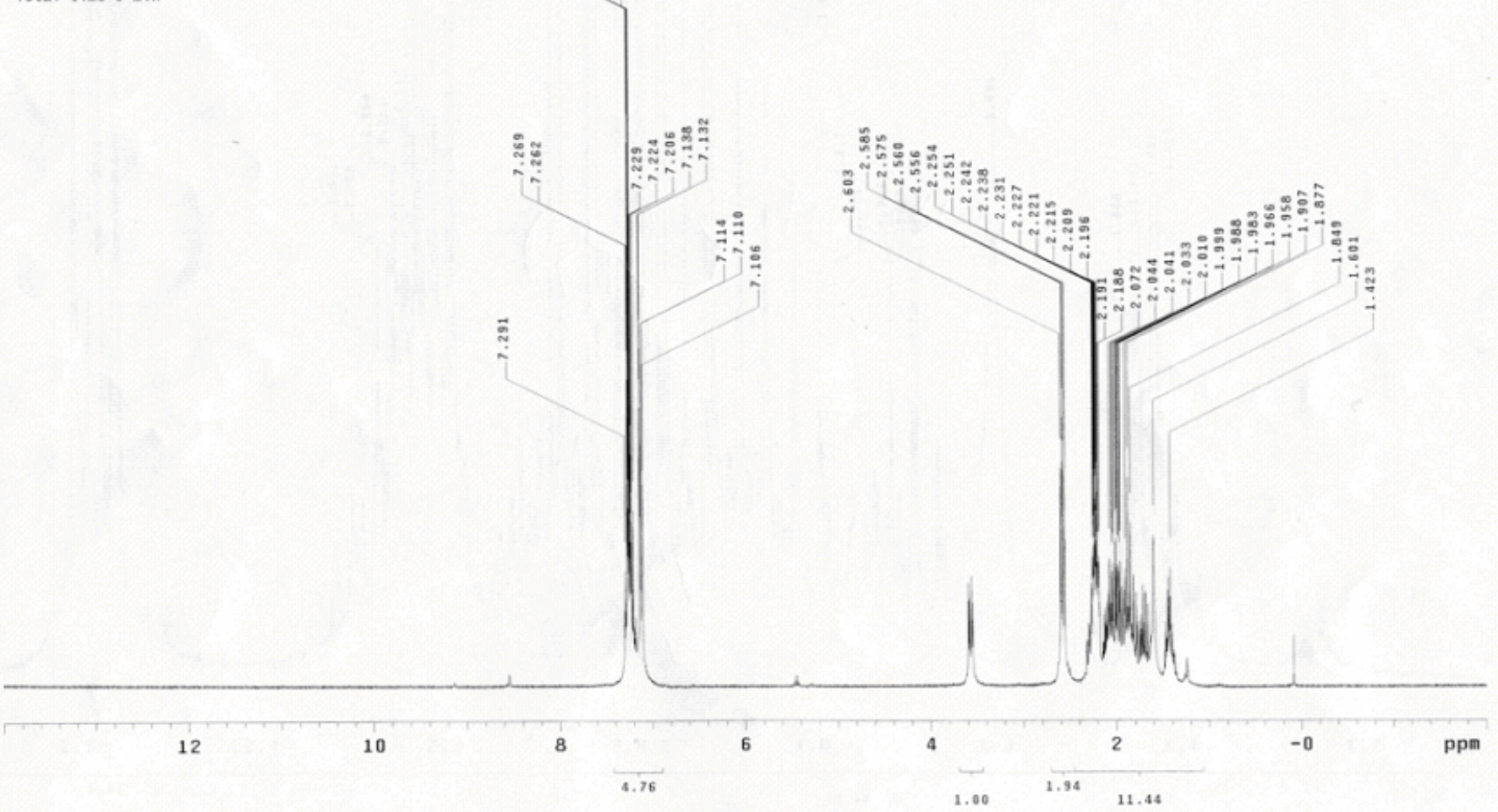




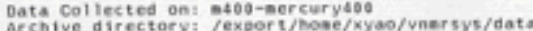

sasple difecto

pulse sequencel szpu

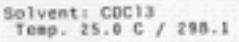

Relax. delay 1,000 sec

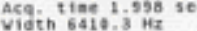

4 repetitions 400.1245562 MH

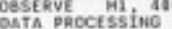

Tsyzectsts
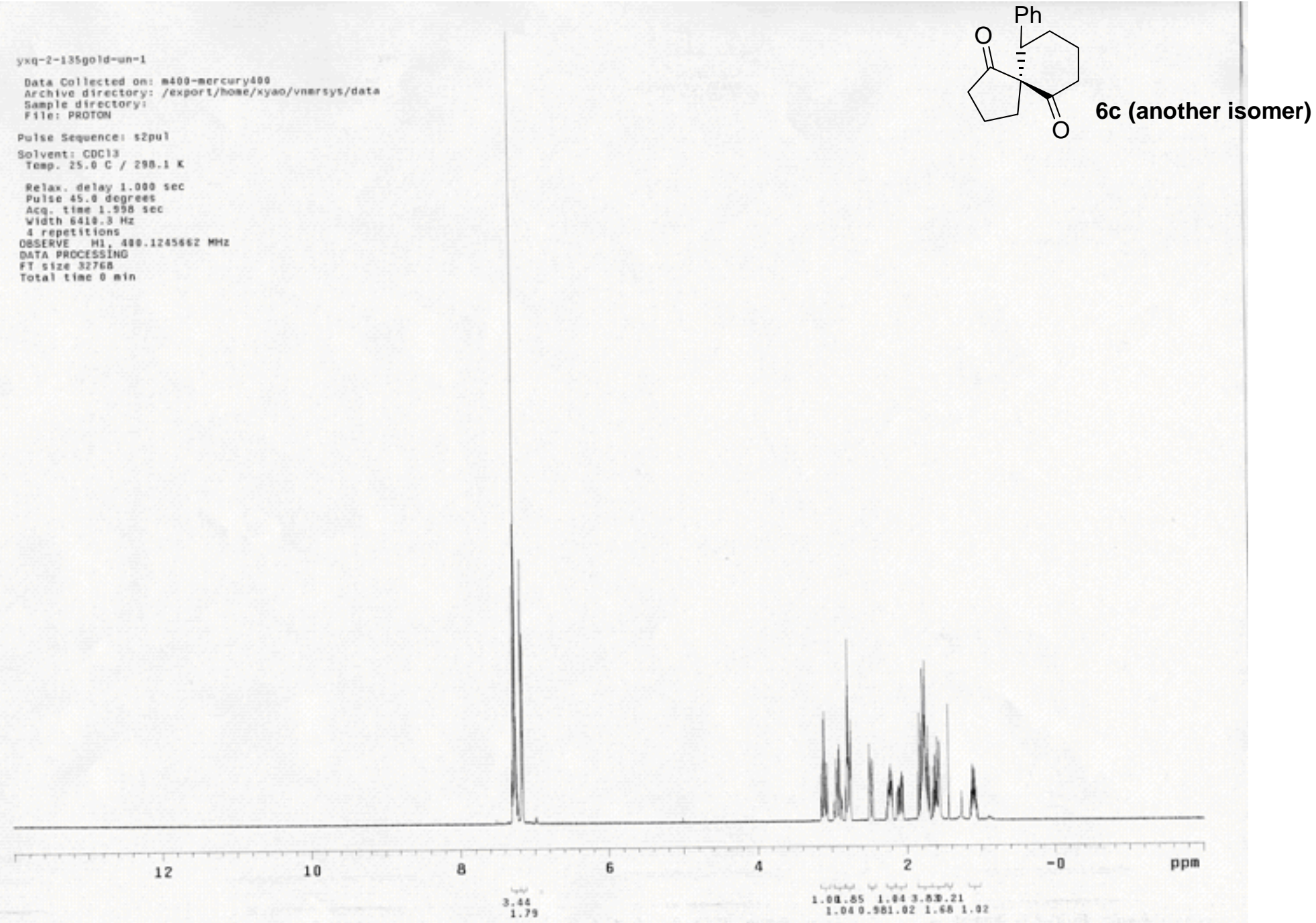
STANDaro IN OBStruve

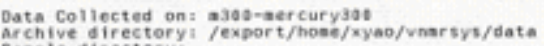

sample directiory

Pulse sequence: s2pul

Solvent: $\mathrm{coc}_{13}$

Relax. delay 1.900 se

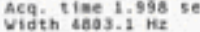

8 repetitions

DATA PROCESSTHO

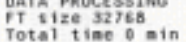

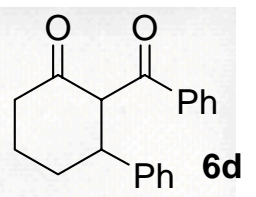




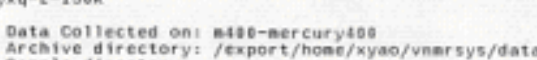

anple direces

pulse sequence: szpul

Solvent: $\mathrm{CeCl}^{2} / 298.1$

Relax. delay 1.1000 sec

Aca

vidin $6410.3 \mathrm{~Hz}$

OESERVE H1. ${ }_{400}$. 1245662 MHI

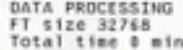
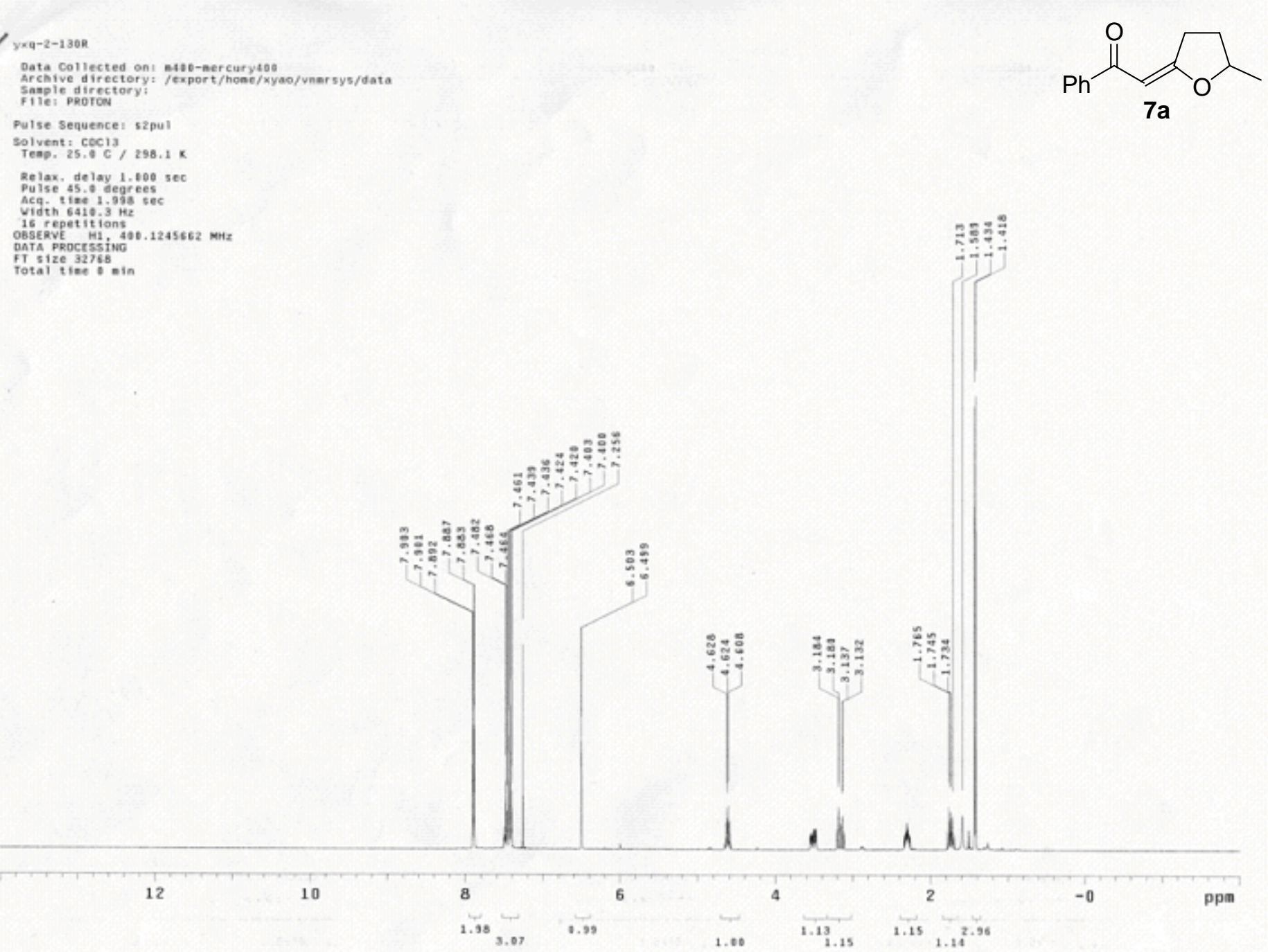
STANOARO 1 H OESERVE

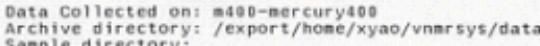
File: pRoron

Pulse Sequence: s2pul

Solvent: $\mathrm{CDCl}^{3}$, $298.1 \mathrm{~K}$
Temp. $25.0 \mathrm{C} / 298.1 \mathrm{C}$

Relax. delay 1,000 sec
Pulse 45.0 degrees

Acgit ${ }^{2}$ ine 1.998 sec

OSSERVE HH 400.1245662 MHZ

DATA PROCESSINU
FT SIZE 32768

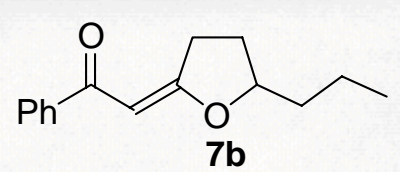

Total time on $\cos$

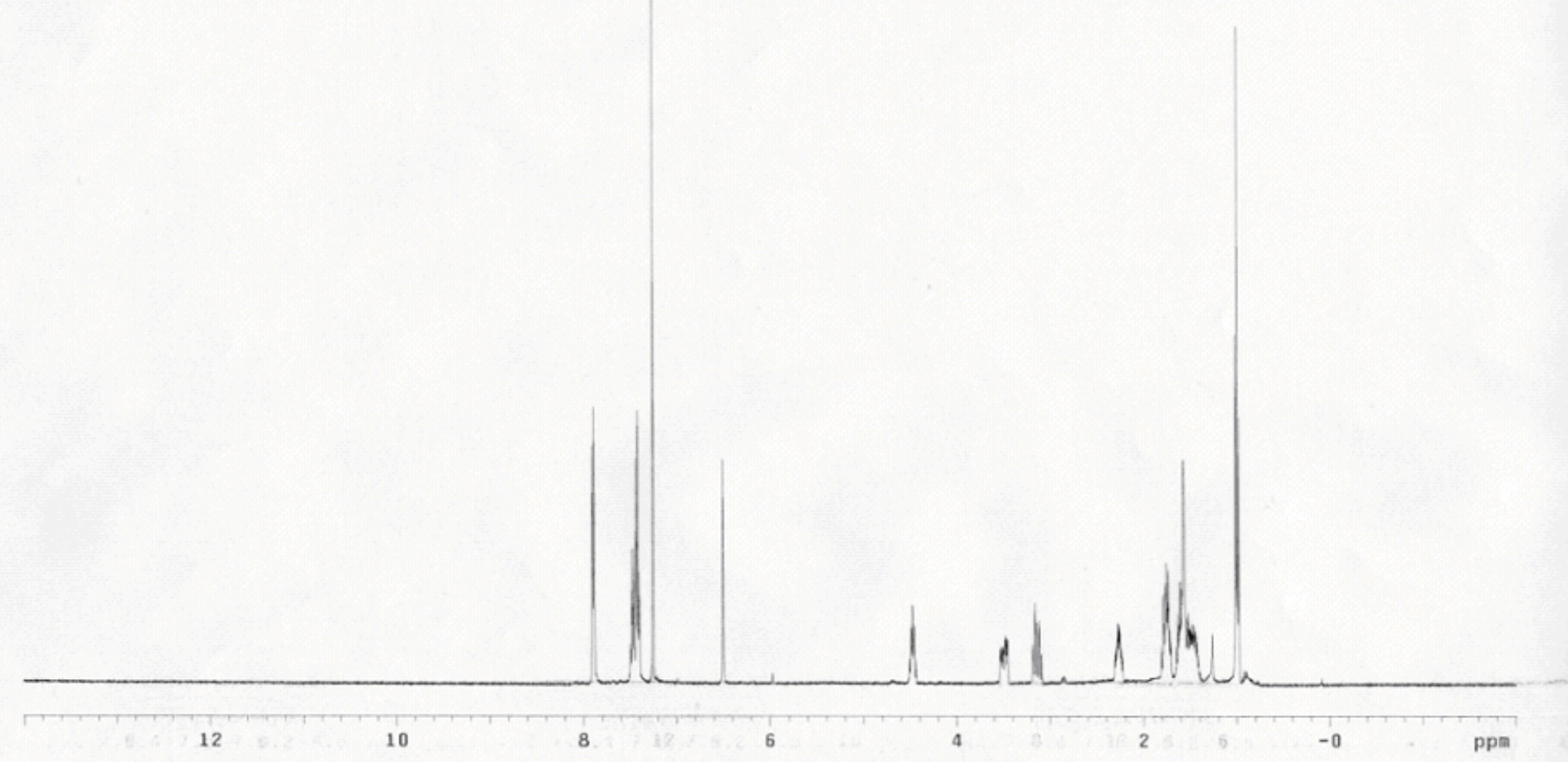

